\title{
Corrigendum to: Risk factors associated with inpatient cardiac arrest during emergency endotracheal intubation at general wards
}

\section{Chul Park}

Division of Pulmonary Medicine, Department of Internal Medicine, Wonkwang University Hospital, Iksan, Korea

Acute and Critical Care 2020;34:212-218.

https://doi.org/10.4266/acc.2019.00598

In the article titled "Risk factors associated with inpatient cardiac arrest during emergency endotracheal intubation at general wards" published on August 2019 [1], there was plagiarism suspicion as the abstract, limitation, and results are quite similar with those in the article by Wardi et al. [2]. In response, the author explained that there are limitations in clinical research, such as the fact that many similar papers exist and that this study was not entirely new ideas, and that the target groups were set differently with retrospective study nature [25]. The author admitted to omitting to cite this paper [2] as a reference in the course of writing the paper. The Editorial Board of Acute and Critical Care decided to publish a corrigendum to correct this plagiarism controversy according to the flowchart of COPE. We would like to apologize for any inconvenience that may have caused.

The revised file can be found on the following page.

\section{REFERENCES}

1. Park C. Risk factors associated with inpatient cardiac arrest during emergency endotracheal intubation at general wards. Acute Crit Care 2019;34:212-8.

2. Wardi G, Villar J, Nguyen T, Vyas A, Pokrajac N, Minokadeh A, et al. Factors and outcomes associated with inpatient cardiac arrest following emergent endotracheal intubation. Resuscitation 2017;121:76-80.

3. Heffner AC, Swords DS, Neale MN, Jones AE. Incidence and factors associated with cardiac arrest complicating emergency airway management. Resuscitation 2013;84:1500-4.

4. Kim WY, Kwak MK, Ko BS, Yoon JC, Sohn CH, Lim KS, et al. Factors associated with the occurrence of cardiac arrest after emergency tracheal intubation in the emergency department. PLoS One 2014;9:e112779.

5. Jaber S, Amraoui J, Lefrant JY, Arich C, Cohendy R, Landreau L, et al. Clinical practice and risk factors for immediate complications of endotracheal intubation in the intensive care unit: a prospective, multiple-center study. Crit Care Med 2006;34:2355-61.

\section{Corrigendum}

Copyright (־) 2020 The Korean Society of Critical Care Medicine

This is an Open Access article distributed under the terms of Creative Attributions Non-Commercial License (https:// creativecommons.org/li-censes/by-nc/4.0/) which permits unrestricted noncommercial use, distribution, and reproduction in any medium, provided the original work is properly cited. 


\section{Risk factors associated with inpatient cardiac arrest during emergency endotracheal intubation at general wards}

\section{Chul Park}

Division of Pulmonary Medicine, Department of Internal Medicine, Wonkwang University Hospital, Iksan, Korea
Background: Peri-intubation cardiac arrest (PICA) is a rare but life-threatening complication. Emergency endotracheal intubation (ETI) can be more complicated at the general ward than in other spaces. Few studies have described PICA at the general ward. This study aimed to identify risk factors associated with PICA at general ward patients.

Methods: We conducted a retrospective observational study of patients at two institutions between January 2016 to December 2017. Inclusion criteria were defined that general ward patients emergently intubated who experienced cardiac arrest within 20 minutes after ETI. The non-PICA group consisted of general ward patients emergently intubated without cardiac arrest.

Results: Fifteen out of 362 patients (3.6\%) presented a PICA that occurred during the study period. All PICA patients had unplanned intensive care unit (ICU) admissions and five of them are dead. Intubation-related shock index, several intubation attempts, pre-ETI vasopressor use, and neuromuscular blocking agent (NMBA) use, especially succinylcholine, were associated with PICA in our study. Clinical outcomes of ICU and hospital length of stay, survival to discharge, and neurologic outcome at hospital discharge were not significantly different between PICA and other types of inpatient cardiac arrest (OTICA).

Conclusion: We identified four independent risk factors for PICA, and pre-intubation hemodynamic stabilization and avoidance of NMBA were possibly modifiable factors of PICA at the general ward. Clinical outcomes of PICA were similar to those of OTICA.

Key Words: endotracheal intubation; heart arrest; neuromuscular blocking agent; risk factor; shock

\section{INTRODUCTION}

Airway management, such as tracheal intubation, is a vital component of emergency medicine. As a means of controlling the airway in emergency settings, rapid sequence intubation (RSI) is widely used as the gold standard technique for tracheal intubation [1,2]. Meanwhile, the safety and effectiveness of tracheal intubation have been well established. However, research on the risks and complications associated with emergency intubation in general ward patients is still obscure $[3,4]$.

Among complications related to intubation including failed intubation, esophageal intu-

\section{Original Article}

Received: June 19, 2019

Revised: August 15, 2019

Accepted: August 20, 2019

Corresponding author

Chul Park

Division of Pulmonary Medicine, Department of Internal Medicine, Wonkwang University Hospital, 895 Muwang-ro, Iksan 54538, Korea

Tel: +82-63-859-2670

Fax: +82-63-855-2025

E-mail:cholssak21@gmail.com

Copyright () 2020 The Korean Society of Critical Care Medicine

This is an Open Access article distributed under the terms of Creative Attributions Non-Commercial License (http:// creativecommons.org/li-censes/by-nc/4.0/) which permits unrestricted noncommercial use, distribution, and reproduction in any medium, provided the original work is properly cited. 
bation, pulmonary aspiration, and hypoxia had been commonly reported. However, there have been few studies of Periintubation cardiac arrest (PICA) [5-7]. The development of post-intubation hypotension is increasingly recognized as a complication independently associated with morbidity and mortality $[8,9]$. Taken together, these facts highlight the need for additional evidence and insights into hemodynamic instability following endotracheal intubation (ETI).

Cardiac arrest is considered one of the most serious complications occurring after intubation. However, very few studies have explored this serious complication. A single-center study reported that PICA occurred in $4 \%$ of all patients in the emergency room and that the in-hospital mortality rate of patients was $84 \%$ even after return of spontaneous circulation (ROSC) [10]. This study aims to identify the prevalence and risk factors associated with PICA in hospitalized patients. We also investigated the differences between PICA and other types of inpatient cardiac arrest (OTICA) in terms of length of hospital stay, in-hospital mortality, and neurological outcomes at hospital discharge.

\section{MATERIALS AND METHODS}

\section{Study Participants}

This is a retrospective observational study conducted at a tertiary referral center and secondary medical center in Jeollabuk-do, South Korea from January 2016 to December 2017, and based on chart review of adult inpatients. The Institutional Review Board approved this study (IRB No. 2019-07-007) and waived the requirement for informed consent because of the observational nature of the study. In addition, patients' information was anonymized and de-identified prior to analysis. All ETIs were carried out by residents or fellows in different medical specialties, such as anesthesia and internal medicine, who had completed advanced resuscitation training courses as part of the advanced cardiac life support program.

Inclusion criteria were as follows: patients over 18 years old and those who developed cardiac arrest during intubation procedure or within 20 minutes of intubation among patients hospitalized in general wards during the study period. Patients were excluded if they developed PICA not in the general ward including Emergency Department, intensive care unit (ICU), and Surgical Ward, if they developed PICA before intubation, or sufficient data was not present before and after intubation.

Tracheal intubation was performed using RSI as the standardized protocol $[2,11]$. In situations requiring intubation, patients were pre-oxygenated in the supine position with a

\section{KEY MESSAGES}

- Risk factors of peri-intubation cardiac arrest for inpatients were intubation-related shock index, number of intubation attempts, pre-endotracheal tube intubation vasopressor use, and neuromuscular blocking agent use.

- Among the four independent risk factors for peri-intubation cardiac arrest, pre-intubation hemodynamic stabilization and avoidance of neuromuscular blocking agent were modifiable to decrease the risk of peri-intubation cardiac arrest.

- Clinical outcomes of intensive care unit and hospital length of stay, survival to discharge, and neurologic outcome at hospital discharge were similar between periintubation cardiac arrest and other types of inpatient cardiac arrest.

head-down tilt. Sedative agents such as benzodiazepine or non-benzodiazepine, and/or analgesics were given, and then subsequently neuromuscular blocking agents were used as part of RSI. ETI was performed by identifying the vocal cords directly or via video laryngoscope. We used succinylcholine as a depolarizing agent (neuromuscular blocker), while cisatracurium, vecuronium, and rocuronium were used during ETI as non-depolarizing agents. If the vocal cord was not visible, manual compression was applied at the cricoid cartilage. In general, $8 \mathrm{~mm}$ endotracheal tubes were used for male patients and $7.5 \mathrm{~mm}$ endotracheal tubes were used for female patients, otherwise practitioners chose an appropriate tube size at their discretion.

\section{Data collection and definitions}

For all patients and controls, we collected the following data from medical records: age, sex, body mass index (BMI), medical history, Sequential Organ Failure Assessment (SOFA) scores on ICU admission [12], and indication for tracheal intubation. Data relating to intubation was collected by an independent observer before or during intubation, include: the number of intubation attempts, duration of intubation, time of intubation (day or night), termination of sedation, use of neuromuscular blocking agents, pre-intubation vital signs, shocking index [10], and use of vasopressors. To compare clinical outcomes between PICA and OTICA, we analyzed the presence of ROSC, ICU, and hospital lengths of stay, survival at hospital discharge, initial cardiac rhythm, and cerebral performance category (CPC) scores at hospital discharge [13].

Intubation duration was defined as a time period from the 
start of sedative administration to the end of intubation. The time of intubation was defined as either day or night according to time of day (7 AM to $11 \mathrm{PM}$ ) and time of night (11 PM to 7 AM) [2]. Cardiac arrest was defined as the delivery of chest compression, the loss of detectable pulse or the use of defibrillation. Pre-ETI vital signs were defined as the minimum systolic or diastolic blood pressure and the maximum heart rate within six hours on the basis of intubation duration. If the minimum blood pressure did not coincide with the maximum heart rate at different time points, the shock index value was calculated for each time point, and the highest value was used. Shock index was defined as last recorded heart rate divided by systolic blood pressure prior to intubation attempt within six hours. Intubation-related shock index was defined as the shock index value multiplied by time of intubation. Neurological outcome was evaluated via CPC scores, where CPC scores of 1 or 2 correspond to good outcome, while CPC scores of 3 to 5 correspond to poor outcome.

\section{Statistical Analysis}

In this study, continuous variables were presented as study mean \pm standard deviation or median and interquartile range, and categorical variables were presented as percentages. An unpaired t-test or Mann-Whitney U-test was used to compare continuous variables between the PICA and non-cardiac arrest group patients who underwent intubation. A chi-square test was used to compare categorical variables between the two groups. Logistic regression analysis was performed to obtain independent variables that have an influence on the development of PICA. Based on results from univariate regression, the variables with less than 0.2 as a P-value were included in a multi-variate analysis. Finally, we followed the backward elimination method to build a multiple regression model for which the value of $\mathrm{P}<0.05$ was considered statistically significant.

\section{RESULTS}

\section{Patient Characteristics}

A total of 362 patients were included in this study as the PICA group $(n=15)$ and non-PICA group $(n=347)$ (Table 1). Compared with the PICA group, the non-PICA group showed younger, male dominant, and lower BMI trends, although there were no significant differences between two groups. Stroke was the most common comorbidity in both groups. However, there were no significant differences in baseline comorbidities between groups. Further, the SOFA scores on ICU admis-
Table 1. Demographics and baseline characteristics of study patients

\begin{tabular}{lccc}
\hline Characteristics & $\begin{array}{c}\text { PICA } \\
(\mathrm{n}=15)\end{array}$ & $\begin{array}{c}\text { Non-PICA } \\
(\mathrm{n}=347)\end{array}$ & P-value \\
\hline Age $(\mathrm{yr})$ & $60.1 \pm 18.9$ & $58.6 \pm 19.1$ & 0.76 \\
Male sex & $8(53.3)$ & $225(64.8)$ & 0.12 \\
BMI $\left(\mathrm{kg} / \mathrm{m}^{2}\right)$ & $21.6 \pm 4.1$ & $22.0 \pm 4.0$ & 0.83 \\
Comorbidity & & & \\
COPD & $1(6.7)$ & $46(13.3)$ & 0.72 \\
Congestive heart failure & $2(13.3)$ & $26(7.5)$ & 0.32 \\
MI & $3(20.0)$ & $40(11.5)$ & 0.40 \\
Stroke & $7(46.7)$ & $172(49.6)$ & 0.85 \\
CKD & $4(26.7)$ & $37(10.7)$ & 0.09 \\
Liver cirrhosis & $2(13.3)$ & $20(5.8)$ & 0.25 \\
SOFA score & $8.0(3.3-12.7)$ & $7.4(3.6-11.2)$ & 0.24 \\
Reasons for intubation & & & 0.15 \\
Acute respiratory failure & $7(46.7)$ & $275(79.3)$ & \\
Altered mental status & $3(20.0)$ & $59(17.0)$ & \\
Shock & $2(13.3)$ & $10(2.9)$ & \\
Others & $3(20.0)$ & $3(0.9)$ & \\
\hline
\end{tabular}

Values are presented as mean \pm standard deviation, number (\%), or median (interquartile range).

PICA: peri-intubation cardiac arrest; BMI: body mass index; COPD: chronic obstructive pulmonary disease; MI: myocardial infarction; CKD: chronic kidney disease; SOFA: Sequential Organ Failure Assessment.

sion were not significantly different between the groups. The indication for tracheal intubation, acute respiratory distress, was the most common in both groups, followed by other causes such as altered level of consciousness and shock states. However, there was no significant indication differences between the groups.

\section{Risk Factors Associated with PICA}

Table 2 presents data collected before and during intubation between the two groups. The comparison between the PICA and non-PICA groups revealed statistically significant differences in the number of intubation attempts, duration of intubation, time of intubation, use of neuromuscular blocking agents, use of succinylcholine, shock index, intubation-related shock index, and pre-ETI vasopressor use. The demand for multiple intubation attempts was higher in the PICA group. Intubation time was also longer in the PICA group (3.5 minutes vs. 2.1 minutes, $\mathrm{P}<0.01$ ). In terms of time of intubation, a higher proportion of intubations were performed at night (73.3\% vs. $16.7 \%, \mathrm{P}<0.01$ ), along with increased use of neuromuscular blocking agents $(60.0 \%$ vs. $38.9 \%, \mathrm{P}=0.02)$ and use 
Table 2. Characteristics of procedure obtained before and during intubation

\begin{tabular}{|c|c|c|c|}
\hline Characteristics & $\begin{array}{c}\text { PICA } \\
(n=15)\end{array}$ & $\begin{array}{l}\text { Non-PICA } \\
(n=347)\end{array}$ & P-value \\
\hline No. of intubation attempts & & & 0.03 \\
\hline 1 & $8(53.3)$ & 309 (89.0) & \\
\hline 2 & $5(33.3)$ & $37(10.7)$ & \\
\hline$\geq 3$ & $2(13.3)$ & $1(0.3)$ & \\
\hline $\begin{array}{l}\text { Intubation time (from induction } \\
\text { to tube insertion, min) }\end{array}$ & $3.5(1.2-5.9)$ & $2.1(1.1-3.2)$ & $<0.01$ \\
\hline Procedure time of day & & & $<0.01$ \\
\hline Daytime (7:00 AM to 10:59 PM) & $4(26.7)$ & $289(83.3)$ & \\
\hline Nighttime (11:00 PM to 6:59 AM) & 11 (73.3) & $58(16.7)$ & \\
\hline Type of sedative agents & & & 0.67 \\
\hline Midazolam & $10(66.7)$ & $302(87.0)$ & \\
\hline Ketamine & $3(20.0)$ & 39 (11.3) & \\
\hline Others or multiple & $2(13.3)$ & $6(1.7)$ & \\
\hline NMBA use for RSI & $9(60.0)$ & 135 (38.9) & 0.02 \\
\hline Use of succinylcholine & $6(40.0)$ & $50(14.4)$ & $<0.01$ \\
\hline \multicolumn{4}{|l|}{ Pre-ETI vital sign } \\
\hline $\mathrm{SBP}(\mathrm{mm} \mathrm{Hg})$ & $118.6 \pm 30.5$ & $125.2 \pm 27.9$ & 0.41 \\
\hline $\mathrm{DBP}(\mathrm{mm} \mathrm{Hg})$ & $67.2 \pm 22.5$ & $68.5 \pm 21.3$ & 0.72 \\
\hline $\mathrm{HR}(/ \min )$ & $112.4 \pm 25.5$ & $102.9 \pm 23.9$ & 0.08 \\
\hline Shock index ${ }^{a}$ & $0.95 \pm 0.32$ & $0.80 \pm 0.25$ & 0.04 \\
\hline Intubation-related shock index & $3.3(1.1-5.6)$ & $1.7(0.9-2.6)$ & $<0.01$ \\
\hline Pre-ETI vasopressor use & $10(66.7)$ & 31 (8.9) & $<0.01$ \\
\hline
\end{tabular}

Values are presented as number (\%), median (interquartile range), or mean \pm standard deviation.

PICA: peri-intubation cardiac arrest; NMBA: neuromuscular blocking agent; RSI: rapid sequence intubation; ETI: endotracheal intubation; SBP: systolic blood pressure; DBP: diastolic blood pressure; HR: heart rate. a'Shock index =HR/SBP; ${ }^{b}$ Intubation-related shock index $=$ shock index $x$ intubation time. of succinylcholine ( $40 \%$ vs. $14.4 \%, \mathrm{P}<0.001$ ) were seen in the PICA group. The values for shock index and intubation-related shock index were significantly higher in the PICA (0.95 vs. $0.80,3.3$ vs. 1.7 ) group. The use of vasopressors prior to intubation was also higher in the PICA group (66.7\% vs. $8.9 \%$ ).

Based on these findings, we performed logistic regression analysis to identify variables associated with PICA (Table 3). The results revealed that the number of intubation attempts (odds ratio [OR], 3.10; 95\% CI, 1.12 to 7.86), use of neuromuscular blocking agents (OR, 1.21; 95\% CI, 1.08 to 3.15), intubation-related shock index (OR, 4.06; 95\% CI, 1.78 to 12.11 ), use of succinylcholine (OR, 3.45; 95\% CI, 1.20 to 5.49), and vasopressor use prior to intubation (OR, 2.69; 95\% CI, 1.15 to 7.42 ) were associated with clinical variables of PICA.

\section{Comparison between PICA and OTICA}

We compared clinical outcomes of PICA and OTICA during the study period (Table 4). A total of 402 inpatients with OTICA were identified. The OTICA group patients were older and demonstrated a greater chance of ROSC and survival to hospital discharge compared to PICA group patients, while there was no significant difference between the two groups. ICU and hospital lengths of stay were longer in the OTICA group. However, once again there was no significant difference between the two groups. Among initial cardiac rhythms, pulseless electrical activity was revealed as the most common rhythm between the two groups, however no significant differences were observed. Neurological outcome at hospital discharge was also similar between the two groups.

Table 3. Univariable and multivariable logistic regression analysis for clinical variables associated with PICA

\begin{tabular}{|c|c|c|c|c|}
\hline \multirow{2}{*}{ Variable } & \multicolumn{2}{|c|}{ Univariable } & \multicolumn{2}{|c|}{ Multivariable } \\
\hline & OR $(95 \% \mathrm{Cl})$ & P-value & OR $(95 \% \mathrm{Cl})$ & P-value \\
\hline Age & $0.97(0.94-1.02)$ & 0.579 & & \\
\hline SOFA score & $0.89(0.77-1.06)$ & 0.151 & & \\
\hline Number of intubation attempts & $4.67(2.35-7.22)$ & 0.014 & $3.10(1.12-7.86)$ & 0.026 \\
\hline Nighttime procedure & $3.21(2.97-4.01)$ & 0.033 & & \\
\hline NMBA use for RSI & $2.34(1.76-3.22)$ & 0.026 & $1.21(1.08-3.15)$ & 0.035 \\
\hline Use of succinylcholine & $4.50(1.45-7.20)$ & $<0.001$ & $3.45(1.20-5.49)$ & 0.005 \\
\hline Shock index & $1.91(1.00-2.52)$ & 0.043 & & \\
\hline Intubation-related shock index & $6.39(2.17-12.44)$ & $<0.001$ & $4.06(1.78-12.11)$ & 0.002 \\
\hline Pre-ETI vasopressor use & $5.67(3.27-8.12)$ & $<0.001$ & $2.69(1.15-7.42)$ & 0.012 \\
\hline
\end{tabular}

PICA: peri-intubation cardiac arrest; OR: odds ratio; Cl: confidence interval; SOFA: Sequential Organ Failure Assessment; NMBA: neuromuscular blocking agent; RSI: rapid sequential intubation; ETI: endotracheal intubation. 
Table 4. Comparison of clinical outcomes between PICA and OTICA

\begin{tabular}{lccc}
\hline Characteristics & $\begin{array}{c}\text { PICA } \\
(n=15)\end{array}$ & $\begin{array}{c}\text { OTICA } \\
(n=402)\end{array}$ & P-value \\
\hline Age (yr) & $60.1 \pm 18.9$ & $64.6 \pm 19.1$ & 0.06 \\
ROSC & $11(73.3)$ & $332(82.6)$ & 0.49 \\
ICU LOS (day) & $20.3 \pm 6.9$ & $23.9 \pm 5.9$ & 0.66 \\
Hospital LOS (day) & $43.6 \pm 12.2$ & $45.1 \pm 13.9$ & 0.72 \\
Survival to hospital discharge & $8(53.3)$ & $284(70.6)$ & 0.08 \\
Initial rhythm of arrest & & & 0.24 \\
PEA & $9(60.0)$ & $299(74.4)$ & \\
VTNF & $3(20.0)$ & $66(16.4)$ & \\
Asystole & $1(6.7)$ & $21(5.2)$ & \\
AF & $1(6.7)$ & $11(2.7)$ & \\
Uncertain & $1(6.7)$ & $5(1.2)$ & \\
Neurologic outcome at hospital discharge & & 0.12 \\
Good (CPC score 1 or 2) & $8(53.3)$ & $270(67.2)$ & \\
Poor (CPC score 3, 4, or 5) & $7(46.7)$ & $14(13.7)$ & \\
\hline
\end{tabular}

Values are presented as mean \pm standard deviation or number (\%). PICA: peri-intubation cardiac arrest; OTICA: other types of inpatient cardiac arrest; ROSC: return of spontaneous circulation; ICU: intensive care unit; LOS: length of stay; PEA: pulseless electrical activity; VT: ventricular tachycardia; VF: ventricular fibrillation; AF: atrial fibrillation; CPC: cerebral performance category.

${ }^{\mathrm{a} G o o d}$ neurologic outcome is CPC score 1 or 2, and poor neurologic outcome is CPC score 3,4 , or 5 . Patients who died are not included in this category.

\section{DISCUSSION}

The present study identified the number of intubation attempts, intubation-related shock index, use of neuromuscular blocking agents, and pre-ETI vasopressor use as factors associated with PICA. Use of neuromuscular blocking agents and hemodynamic instability prior to intubation, measured by intubation-related shock index and pre-ETI vasopressor use required particular attention, because these variables were modifiable. The comparison between PICA and OTICA revealed that there were no significant differences in ICU or hospital length of stay, survival at hospital discharge, or clinical and neurological outcomes.

Previous studies have reported several mechanisms involving PICA in Emergency Department settings [14-17], describing that cardiac arrest patients may not tolerate decreased preload and reduction of venous return, which are triggered by an increase in intrathoracic pressure when positive pressure ventilation is applied in patients with shock after intubation [18]. It can also result from physiological changes due to severe diseases such as acidosis, which can be exacerbated upon respiratory compensation being compromised when respiratory rate and effective respiratory response are negatively affected during intubation. In addition, the use of sedatives and neuromuscular blocking agents may lead to cardiac arrest due to their inhibitory action against catecholamine secretion stimulated in response to stress. In light of this view, pre-intubation hemodynamic instability, once confirmed, may reduce cardiac output during intubation and attenuate respiratory compensation in proportion to intubation time, leading to the deterioration of acidosis. Hemodynamic instability is also the main reason why the inhibition of neuromuscular blocking agents upon catecholamine secretion potentially causes cardiac arrest [17-23].

The intubation-related shock index, identified as one of risk factors of PICA in this study, was used as a measure of hemodynamic instability and intubation duration. Several studies have reported shock index as an independent predictor of PICA in Emergency Department patients and to emphasize the importance of intervention necessary to prevent cardiac arrest $[18,24]$. Further studies have been conducted to explore the index in ICU settings and to develop interventions effective in preventing complications related to ETI. As a result, a study reported significantly decreased intubation-related complications, including PICA, after implementing intervention measures in which a checklist was prepared to keep track of all intravenous fluids or conscious intubation [25]. In the event of cardiac arrest in an Emergency Department or ICU, it is possible to take immediate actions, whereas rapid response to cardiac arrest on general wards becomes difficult due to the lack of monitoring facilities and attending medical personnel, understaffing, or restrictions on medication for intervention $[18,26]$. Therefore, the results of this study are expected to be clinically useful to improve procedures for PICA prevention within general wards when emergency intubation is necessary.

This study has several limitations. First, given its observational study nature, the possibility of selection bias and presence of confounding factors cannot be eliminated in our findings. The major findings of our study represent associations between variables, not cause-effect relationships. All hemodynamic variables including vital signs, are not standardized. Blood pressure records indicate values measured intermittently using a non-invasive method. Therefore, the values need to be interpreted with caution. Second, as we evaluated a short period of clinical data before intubation procedure, outcomes might be impacted by additional factors or variables. Finally, the fact that data were collected at the two hospitals limited 
the analysis of differences between patient characteristics from each hospital and the factors influencing protocols used in emergency situations. Moreover, it remains uncertain whether the results of this study conducted in a provincial city can be generalized to PICA patients in clinical settings in other larger or in more urban areas.

Despite these limitations, this study provides initial insight into the factors associated with the development and prevalence of PICA on general wards and suggests a new concept of intubation-related shock index, which has a greater explanatory power than the current shock index. Further prospective and multi-center studies are needed to identify modifiable risk factors for PICA prevention in a larger patient population with adequately controlled confounding factors.

\section{CONFLICT OF INTEREST}

No potential conflict of interest relevant to this article was reported.

\section{ORCID}

Chul Park https://orcid.org/0000-0002-6031-009X

\section{REFERENCES}

1. Stollings JL, Diedrich DA, Oyen LJ, Brown DR. Rapid-sequence intubation: a review of the process and considerations when choosing medications. Ann Pharmacother 2014;48:62-76.

2. Hinkelbein J, Kranke P. Rapid sequence induction. Anasthesiol Intensivmed Notfallmed Schmerzther 2018;53:631-4.

3. Andersen LW, Granfeldt A, Callaway CW, Bradley SM, Soar J, Nolan JP, et al. Association between tracheal intubation during adult in-hospital cardiac arrest and survival. JAMA 2017; 317:494-506.

4. Izawa J, Komukai S, Gibo K, Okubo M, Kiyohara K, Nishiyama $\mathrm{C}$, et al. Pre-hospital advanced airway management for adults with out-of-hospital cardiac arrest: nationwide cohort study. BMJ 2019;364:1430.

5. Griesdale DE, Bosma TL, Kurth T, Isac G, Chittock DR. Complications of endotracheal intubation in the critically ill. Intensive Care Med 2008;34:1835-42.

6. Schwartz DE, Matthay MA, Cohen NH. Death and other complications of emergency airway management in critically ill adults: a prospective investigation of 297 tracheal intubations. Anesthesiology 1995;82:367-76.

7. Mort TC. Unplanned tracheal extubation outside the operat- ing room: a quality improvement audit of hemodynamic and tracheal airway complications associated with emergency tracheal reintubation. Anesth Analg 1998;86:1171-6.

8. Smischney NJ, Demirci O, Ricter BD, Hoeft CC, Johnson LM, Ansar S, et al. Vasopressor use as a surrogate for post-intubation hemodynamic instability is associated with in-hospital and 90-day mortality: a retrospective cohort study. BMC Res Notes 2015;8:445.

9. Trivedi S, Demirci O, Arteaga G, Kashyap R, Smischney NJ. Evaluation of preintubation shock index and modified shock index as predictors of postintubation hypotension and other short-term outcomes. J Crit Care 2015;30:861.e1-7.

10. Heffner AC, Swords DS, Neale MN, Jones AE. Incidence and factors associated with cardiac arrest complicating emergency airway management. Resuscitation 2013;84:1500-4.

11. Weingart SD, Levitan RM. Preoxygenation and prevention of desaturation during emergency airway management. Ann Emerg Med 2012;59:165-75.e1.

12. Vincent JL, Moreno R, Takala J, Willatts S, De Mendonça A, Bruining H, et al. The SOFA (Sepsis-related Organ Failure Assessment) score to describe organ dysfunction/failure: on behalf of the Working Group on Sepsis-Related Problems of the European Society of Intensive Care Medicine. Intensive Care Med 1996;22:707-10.

13. Phelps R, Dumas F, Maynard C, Silver J, Rea T. Cerebral performance category and long-term prognosis following out-ofhospital cardiac arrest. Crit Care Med 2013;41:1252-7.

14. Camarata SJ, Weil MH, Hanashiro PK, Shubin H. Cardiac arrest in the critically ill. I: A study of predisposing causes in 132 patients. Circulation 1971;44:688-95.

15. Smischney NJ, Seisa MO, Heise KJ, Wiegand RA, Busack KD, Deangelis JL, et al. Predictors of hemodynamic derangement during intubation in the critically ill: a nested case-control study of hemodynamic management-part II. J Crit Care 2018; 44:179-84.

16. Green RS, Turgeon AF, McIntyre LA, Fox-Robichaud AE, Fergusson DA, Doucette S, et al. Postintubation hypotension in intensive care unit patients: a multicenter cohort study. J Crit Care 2015;30:1055-60.

17. Jaber S, Amraoui J, Lefrant JY, Arich C, Cohendy R, Landreau $\mathrm{L}$, et al. Clinical practice and risk factors for immediate complications of endotracheal intubation in the intensive care unit: a prospective, multiple-center study. Crit Care Med 2006;34: 2355-61.

18. Wardi G, Villar J, Nguyen T, Vyas A, Pokrajac N, Minokadeh A, et al. Factors and outcomes associated with inpatient cardiac arrest following emergent endotracheal intubation. Resusci- 
tation 2017;121:76-80.

19. Hasegawa K, Shigemitsu K, Hagiwara Y, Chiba T, Watase H, Brown CA 3rd, et al. Association between repeated intubation attempts and adverse events in emergency departments: an analysis of a multicenter prospective observational study. Ann Emerg Med 2012;60:749-54.e2.

20. Kim WY, Kwak MK, Ko BS, Yoon JC, Sohn CH, Lim KS, et al. Factors associated with the occurrence of cardiac arrest after emergency tracheal intubation in the emergency department. PLoS One 2014;9:e112779.

21. Smischney NJ, Demirci O, Diedrich DA, Barbara DW, Sandefur BJ, Trivedi S, et al. Incidence of and risk factors for post-intubation hypotension in the critically ill. Med Sci Monit 2016; 22:346-55.

22. Marin J, Davison D, Pourmand A. Emergent endotracheal intubation associated cardiac arrest, risks, and emergency implications. J Anesth 2019;33:454-62.
23. Lundstrøm LH, Duez CH, Nørskov AK, Rosenstock CV, Thomsen JL, Møller AM, et al. Avoidance versus use of neuromuscular blocking agents for improving conditions during tracheal intubation or direct laryngoscopy in adults and adolescents. Cochrane Database Syst Rev 2017;5:CD009237.

24. Althunayyan SM. Shock index as a predictor of post-intubation hypotension and cardiac arrest: a review of the current evidence. Bull Emerg Trauma 2019;7:21-7.

25. Jaber S, Jung B, Corne P, Sebbane M, Muller L, Chanques G, et al. An intervention to decrease complications related to endotracheal intubation in the intensive care unit: a prospective, multiple-center study. Intensive Care Med 2010;36:24855.

26. Green R, Hutton B, Lorette J, Bleskie D, McIntyre L, Fergusson D. Incidence of postintubation hemodynamic instability associated with emergent intubations performed outside the operating room: a systematic review. CJEM 2014;16:69-79. 